\title{
ELECTRON ENERGY DISTRIBUTIONS OF AGNS IN THE THIN SYNCHROTRON LIMIT. II. PEAKED ELECTRON ENERGY SPECTRA OF BLAZARS AND OVV QUASARS
}

\author{
IRENE CRUZ-GONZÁLEZ, LUIS SALAS and LUIS CARRASCO \\ Instituto de Astronomía UNAM, Apdo. 70-264, Mexico DF 04510, Mexico
}

\begin{abstract}
.
We analyse multifrequency quasi-simultaneous observations of AGNs with the Inverse Synchrotron Transform (IST) method described in Salas et al. (1993) and in Paper I (this conference).

The observational spectral energy distribution of blazars and OVV quasars used as applications of the IST method were obtained from multifrequency quasi-simultaneous observations of Landau et al. (1986) and Brown et al. (1989). The application of the method requires good spectral coverage, since the presence of gaps larger than $\approx 0.75 d e x$ can introduce ficticious peaks. We present the analysis for: $1749+096,0735+178,1308+326,3 C 273,3 C 345,3 C 279$, OJ287, OJ049, 3C216, 4C39.25.

The observed spectra are transformed to the source rest frame, the inverse transform is calculated over the whole spectrum to derive the electron energy spectra, which gives the amount of energy emitted in each characteristic frequency. Our results show that the electron energy spectra, $\Psi\left(\nu_{c}\right)$, of blazars and OVV quasars consists mainly of 4 or 5 well defined peaks superimposed on a down curved continuum. This indicates that the source radiates mainly around certain values of the electron's critical frequency (or energy, since $\nu_{c} \propto B E^{2}$ ). These peaks are consistent with injection of fresh relativistic particles, occuring in pulses of quasi-monoenergetic groups.

The separation between successive peaks $(\Delta p)$ is $\approx 1.2$ dex in critical frequency, and is characteristic for each object. The peaks in the radio region contain approximately the same number of electrons, consistent with evolution given by the continuity equation for constant $B$. While in the OIR region, the energy radiated increases towards lower frequencies.

A comparison of $\Delta p$ with $L_{b o l}$ and $L_{\text {radio, }}$ and jet parameters such as, jet velocity and $B_{1 p c}$ (inferred from the relativistic jet models of Blandford and Königl 1979) given by Cruz-González and Carrillo (1991), shows that $\Delta p$ increases with jet velocity, stronger $B$ fields imply smaller $\Delta p$, and that it is not correlated with $L_{b o l}$, but is apparently correlated with $L_{\text {radio. }}$

The Inverse Synchrotron Transform method applied in this paper is a powerful tool in the analysis of spectral energy distributions of AGNs.
\end{abstract}

Key words: active galactic nuclei, multiwavelength emission, emission processes

\section{References}

Biretta,J.A., Moore,R.L., Cohen,M.H., 1986, Astrophysical Journal308, 93.

Brown, L.M.J. et al.: 1989, Astrophysical Journal340, 129.

Cruz-González,I., Carrillo,R., 1991, Rev.Mex.Astron.Astrofis 22, 217.

Landau et al.: 1986, Astrophysical Journal308, 78.

Salas, L., Cruz-González, I., Carrasco, L. 1993, in preparation.

T. J.-L. Courvoisier and A. Blecha: Multi-Wavelength Continuum Emission of AGN, 320.

(c) 1994 IAU. Printed in the Netherlands. 